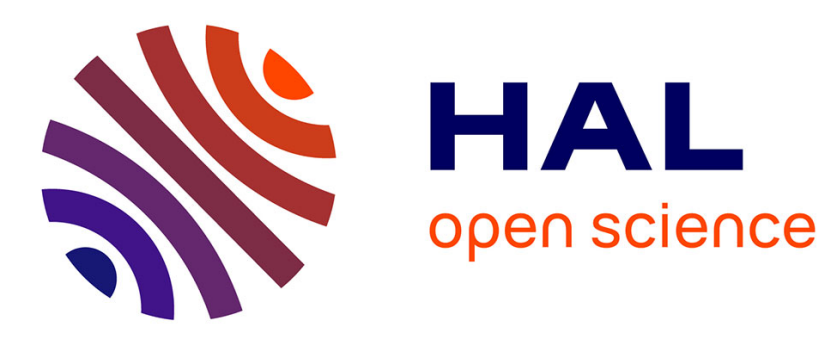

\title{
Stabilization of continuous-time singularly perturbed switched systems
}

\author{
Ivan Malloci, Jamal Daafouz, Claude Iung
}

\section{To cite this version:}

Ivan Malloci, Jamal Daafouz, Claude Iung. Stabilization of continuous-time singularly perturbed switched systems. 48th IEEE Conference on Decision and Control, CDC'09, Dec 2009, Shanghai, China. pp.CDROM. hal-00439265

\section{HAL Id: hal-00439265 \\ https://hal.science/hal-00439265}

Submitted on 7 Dec 2009

HAL is a multi-disciplinary open access archive for the deposit and dissemination of scientific research documents, whether they are published or not. The documents may come from teaching and research institutions in France or abroad, or from public or private research centers.
L'archive ouverte pluridisciplinaire HAL, est destinée au dépôt et à la diffusion de documents scientifiques de niveau recherche, publiés ou non, émanant des établissements d'enseignement et de recherche français ou étrangers, des laboratoires publics ou privés. 


\title{
Stabilization of Continuous-Time Singularly Perturbed Switched Systems
}

\author{
I. Malloci, J. DaAfouz, Member, IEEE, C. Iung, Member, IEEE
}

\begin{abstract}
In this article, stability of continuous-time switched linear systems in the singular perturbation form is investigated. We show that the stability of slow and fast switched subsystems is not a sufficient condition for stability of the corresponding two-time scale switched system, under an arbitrary switching law. Thus, LMI conditions to design a statefeedback control law stabilizing continuous-time singularly perturbed switched linear systems are proposed.
\end{abstract}

\section{INTRODUCTION}

A switched system consists of a set of differential equations, where each equation defines the behavior of a subsystem, and of a law governing the switchings among the subsystems. In the control system framework, one of most studied properties of switched systems is stability [2], [3], [4], [10], [15]. When the switching law is arbitrary, there exist two main approaches to assess system stability. The first one consists in assuming that there is a minimal interval of time between two successive switchings, called dwell time, which ensures the stability [6], [13], [16]. If no assumption is made on the dwell time, the existence of a common Lyapunov function for all the subsystems is required [10], [15].

In practice, many systems involve dynamics operating on two or more time scales [14]. In this case, standard control techniques lead to ill-conditioning problems. Singular perturbation methods can be used to avoid such numerical problems [8], [9]. They consist in decomposing the system into several subsystems, one for each time scale. Thus, a different controller is designed for each of them. In [5], a LMI solution for the linear quadratic optimal control design is proposed for continuous-time singularly perturbed linear systems.

Multiple time scale switched systems are of practical interest in many applications. An example is given by the tail end phase of the rolling process in a hot strip mill, which has been described in [12]. However, these dynamical systems have been the subject of few investigations and, at the moment, the only work addressing two-time scale switched systems is proposed in [1], where the dwell time approach is extended to singularly perturbed continuous-time switched systems with time delay. Classical solutions for LTI singularly perturbed systems are based on the fact that slow and fast dynamics can be considered as decoupled.

This work has been supported by grants from "la région Lorraine, France" and ArcelorMittal Maizières Research.

I. Malloci, J. Daafouz and C. Iung are with the Centre de Recherche en Automatique de Nancy, UMR 7039 CNRS - Nancy Université, ENSEM, 2, Avenue de la forêt de Haye 54516 Vandoeuvre-lès-Nancy, France. Corresponding author: Jamal.Daafouz@ensem.inpl-nancy.fr.
Thus, asymptotic stability of slow and fast manifolds is a sufficient condition for the stability of the two-time scale system. In this article, we will show that, if no assumption on the minimal dwell is made, stability of slow and fast switched subsystems is not a sufficient condition for stability of a two-time scale switched system. An additional constraint taking into account the coupling between slow and fast manifolds has to be considered. We propose LMI conditions, independently of the singular parameter $\varepsilon$, for stability analysis and feedback control design of continuoustime singularly perturbed switched linear systems. These conditions express the fact that a coupling constraint has to be satisfied, in addition to stability of slow and fast switched subsystems, as far as arbitrary switchings may arise. To our knowledge, this is the first work which points out explicitly the fact that asymptotic stability of slow and fast switched subsystems is not sufficient for asymptotic stability of a twotime scale switched system, under an arbitrary switching rule, and which provides a stabilizing control law for this kind of systems.

The article is organized as follows. In section II, switched systems in the singular perturbation form are introduced. In section III, stability problems arising in singularly perturbed systems when arbitrary switchings occur are discussed. In section IV and V, stability analysis and stabilization of continuous singularly perturbed switched linear systems are studied. In section VI, a numerical example is presented.

\section{PRELIMINARIES}

Consider the continuous-time switched system in the singular perturbation form:

$$
\dot{x}(t)=M^{\sigma(t)}(\varepsilon) x(t)+N^{\sigma(t)}(\varepsilon) u(t)
$$

where $\varepsilon>0$ is a small scalar parameter, $x \in \mathbb{R}^{n}$ is the state vector, which is assumed to be available for direct measurement, $u \in \mathbb{R}^{r}$ is the control signal, $\left\{\left(M^{i}(\varepsilon), N^{i}(\varepsilon)\right): i \in\right.$ $\Xi=\{1, \ldots, \mathcal{N}\}\}$ is a family of matrices and $\sigma(t): \mathbb{N} \rightarrow \Xi$ is the switching signal, which is assumed to be unknown a priori. If we assume that the slow and the fast variables are the same for each subsystem $i \in \Xi$, we can write:

$$
x(t)=\left[\begin{array}{l}
x_{1}(t) \\
x_{2}(t)
\end{array}\right],
$$

where $x_{1} \in \mathbb{R}^{n_{1}}$ is the state corresponding to the fast dynamics and $x_{2} \in \mathbb{R}^{n_{2}}$ is the state corresponding to the 
slow dynamics, and

$$
\begin{aligned}
& M^{i}(\varepsilon)=\left[\begin{array}{cc}
\varepsilon^{-1} I_{n_{1}} & 0 \\
0 & I_{n_{2}}
\end{array}\right]\left[\begin{array}{ll}
M_{11}^{i} & M_{12}^{i} \\
M_{21}^{i} & M_{22}^{i}
\end{array}\right], \\
& N^{i}(\varepsilon)=\left[\begin{array}{cc}
\varepsilon^{-1} I_{n_{1}} & 0 \\
0 & I_{n_{2}}
\end{array}\right]\left[\begin{array}{l}
N_{1}^{i} \\
N_{2}^{i}
\end{array}\right],
\end{aligned}
$$

with $M_{11}^{i}$ non-singular matrix for any $i \in \Xi$. Each mode $i$ can be written as

$$
\left\{\begin{array}{l}
\varepsilon \dot{x}_{1}(t)=M_{11}^{i} x_{1}(t)+M_{12}^{i} x_{2}(t)+N_{1}^{i} u(t) \\
\dot{x}_{2}(t)=M_{21}^{i} x_{1}(t)+M_{22}^{i} x_{2}(t)+N_{2}^{i} u(t)
\end{array}\right.
$$

The slow subsystem is:

$$
\dot{x}_{s}(t)=M_{s}^{i} x_{s}(t)+N_{s}^{i} u_{s}(t)
$$

with

$$
\begin{aligned}
& M_{s}^{i}=M_{22}^{i}-M_{21}^{i} M_{11}^{i}{ }^{-1} M_{12}^{i} \\
& N_{s}^{i}=N_{2}^{i}-M_{21}^{i} M_{11}^{i}{ }^{-1} N_{1}^{i} .
\end{aligned}
$$

The fast subsystem is:

$$
\varepsilon \dot{x}_{f}(t)=M_{11}^{i} x_{f}(t)+N_{1}^{i} u_{f}(t) .
$$

The pairs $\left(M_{s}^{i}, N_{s}^{i}\right)$ and $\left(M_{11}^{i}, N_{1}^{i}\right)$ are assumed to be stabilizable in the continuous-time sense, for any $i \in \Xi$. This means that each eigenvalue of $M_{s}^{i}$ or $M_{11}^{i}$ which is in the right-half complex plane is controllable.

\section{MOTIVATION}

Recall that for a continuous-time LTI system in the singular perturbation form

$$
\dot{x}(t)=M(\varepsilon) x(t)
$$

with

$$
M(\varepsilon)=\left[\begin{array}{cc}
\varepsilon^{-1} I_{n_{1}} & 0 \\
0 & I_{n_{2}}
\end{array}\right]\left[\begin{array}{ll}
M_{11} & M_{12} \\
M_{21} & M_{22}
\end{array}\right],
$$

the fast and slow dynamics can be decoupled using the transformation [7]:

$$
\left[\begin{array}{l}
x_{f}(t) \\
x_{s}(t)
\end{array}\right]=\left[\begin{array}{cc}
I_{n_{1}} & L(\varepsilon) \\
-\varepsilon H(\varepsilon) & I_{n_{2}}-\varepsilon H(\varepsilon) L(\varepsilon)
\end{array}\right]\left[\begin{array}{l}
x_{1}(t) \\
x_{2}(t)
\end{array}\right]
$$

with

$$
\begin{aligned}
& M_{12}-M_{11} L(\varepsilon)+\varepsilon L(\varepsilon)\left(M_{22}-M_{21} L(\varepsilon)\right)=0, \\
& M_{21}-H(\varepsilon) M_{11}+\varepsilon\left(M_{22}-M_{21} L(\varepsilon)\right) H(\varepsilon) \\
& -\varepsilon H(\varepsilon) L(\varepsilon) M_{21}=0 .
\end{aligned}
$$

Consider a scalar $\varepsilon_{\max }>0$. For $\varepsilon \in\left(0, \varepsilon_{\max }\right]$, the nonsymmetric algebraic Riccati equation (11) and the Sylvester equation (12) admit the approximated solution $L(\varepsilon)=$ $M_{11}^{-1} M_{12}+O(\varepsilon), H(\varepsilon)=M_{21} M_{11}^{-1}+O(\varepsilon)$. The transformation (10) leads to the following decoupled system:

$$
\left\{\begin{array}{l}
\varepsilon \dot{x}_{f}(t)=\left(M_{11}+O(\varepsilon)\right) x_{f}(t) \\
\dot{x}_{s}(t)=\left(M_{s}+O(\varepsilon)\right) x_{s}(t) .
\end{array}\right.
$$

Hence, asymptotic stability of slow and fast manifolds (i.e. matrices $M_{s}$ and $M_{11}$ are Hurwitz) implies asymptotic stability of the two-time scale system (8) for any $\varepsilon \in\left(0, \varepsilon_{\max }\right]$.
For two-time scale switched systems, this property does not hold, unless dwell time constraints are imposed [1].

However, for an arbitrary switching law, which is the case considered here, a two-time scale switched system can be unstable for any small value of $\varepsilon>0$, even if slow and fast switched manifolds are asymptotically stable. The interpretation of this phenomenon is that for any fixed $\varepsilon>0$, one may exhibit a switching law with a sufficiently high switching frequency which destabilizes the two-time scale switched system, even if the slow and fast switched subsystems are asymptotically stable. To illustrate this fact, let us consider the following example:

$$
\dot{x}(t)=M^{\sigma(t)}(\varepsilon) x(t),
$$

with $\Xi=\{1,2\}$ and

$$
\begin{aligned}
& M_{11}^{1}=\left[\begin{array}{cc}
0 & 1 \\
-3 & -5
\end{array}\right], M_{12}^{1}=\left[\begin{array}{cc}
0 & 0 \\
-1.3 & -0.46
\end{array}\right], \\
& M_{21}^{1}=\left[\begin{array}{cc}
20 & 10 \\
6 & 5
\end{array}\right], \quad M_{22}^{1}=\left[\begin{array}{ll}
0 & 1 \\
2 & 0
\end{array}\right], \\
& M_{11}^{2}=\left[\begin{array}{cc}
0 & 1 \\
-3.8 & -6
\end{array}\right], M_{12}^{2}=\left[\begin{array}{cc}
1 & 5 \\
151 & 49.6
\end{array}\right], \\
& M_{21}^{2}=\left[\begin{array}{cc}
0 & 0 \\
-0.3 & -0.2
\end{array}\right], \quad M_{22}^{2}=\left[\begin{array}{cc}
1 & 0.7 \\
0 & 2
\end{array}\right] .
\end{aligned}
$$

The system (14) has a two-time scale dynamics and matrices $M^{i}(\varepsilon)$ are Hurwitz for any $i \in \Xi$ and for any $\varepsilon \in\left(0, \varepsilon_{\text {max }}\right]$, with $\varepsilon_{\max }=10^{-2}$. Nevertheless, asymptotic stability of each subsystem $i \in \Xi$ is not a sufficient condition for the stability of a switched systems, when the switching law $\sigma(t)$ is arbitrary [10]. Let the fast switched subsystem

$$
\varepsilon \dot{x_{f}}(t)=M_{11}^{\sigma(t)} x_{f}(t)
$$

and the slow switched subsystem

$$
\dot{x_{s}}(t)=M_{s}^{\sigma(t)} x_{s}(t) \text {. }
$$

The sufficient stability condition $M_{11}^{i} P_{f}+P_{f} M_{11}^{i^{\prime}} \prec 0$ of the fast switched subsystem (16) is verified by the Lyapunov matrix $P_{f}=\left[\begin{array}{cc}2 & -1 \\ -1 & 1\end{array}\right] \succ 0$, for any $i \in \Xi$. Also, the sufficient stability condition $M_{s}^{i} P_{s}+P_{s} M_{s}^{i^{\prime}} \prec 0$ of the slow switched subsystem (17) is verified by the Lyapunov matrix $P_{s}=\left[\begin{array}{cc}0.15 & -0.3 \\ -0.3 & 1.36\end{array}\right] \succ 0$, for any $i \in \Xi$.

To show that asymptotic stability of the fast switched subsystem (16) and of the slow switched subsystem (17) does not imply asymptotic stability of the two-time scale switched system (14), consider $\varepsilon=10^{-3}$ and the initial condition $x(0)=\left[\begin{array}{llll}0 & 0 & 1 & 1\end{array}\right]^{\prime}$, with $x(t)=$ $\left[\begin{array}{llll}x_{11}(t) & x_{12}(t) & x_{21}(t) & x_{22}(t)\end{array}\right]^{\prime}$. Switching among the subsystems of (14) each $30 \mathrm{msec}$, we obtain an unstable behavior. Fig. 1 and 2 show the "explosion" of the fast and the slow state variables, respectively. This behavior does not depend on the value of $\varepsilon$ : a switching law $\sigma(t)$ which destabilizes the switched system (14)-(15) can be found for any $\varepsilon \in\left(0, \varepsilon_{\max }\right]$. However, if the switchings among the subsystems of (14) are enough slow to respect 


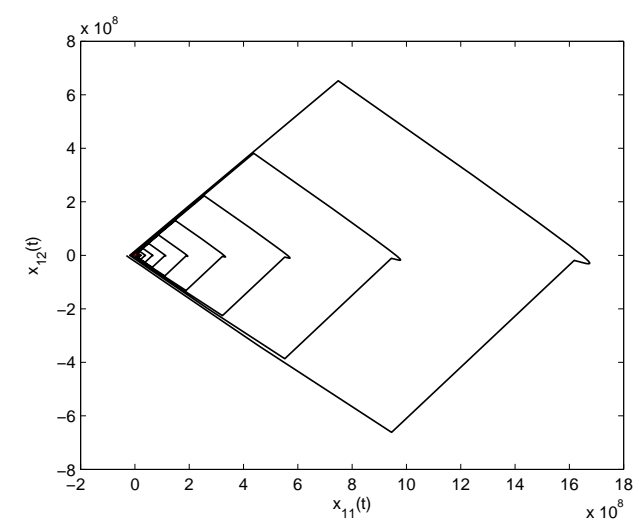

Fig. 1. Slow variables evolution of the system (14) with destabilizing $\sigma(t)$

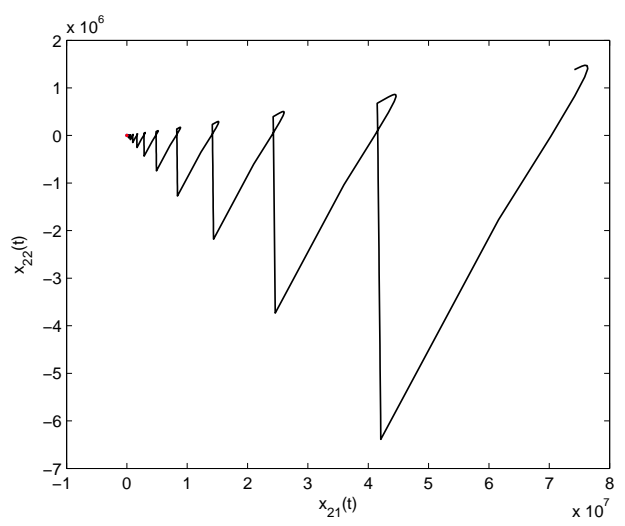

Fig. 2. Fast variables evolution of the system (14) with destabilizing $\sigma(t)$

dwell time conditions, the two-time scale switched system is asymptotically stable. The convergence to zero of the state variables is shown in Fig. 3 and 4 for a dwell time of $150 \mathrm{msec}$. Also the state evolution of each subsystem without switchings is given (dashed and dotted line for the subsystem 1 and 2 , respectively).

The main objective of this article is to provide LMI stability conditions for linear switched systems in the singular perturbation form with an arbitrary switching law. We will show that this corresponds to verify the stability conditions of the slow and the fast switched subsystems and an additional constraint.

\section{STABILITY ANALYSIS}

Consider the autonomous linear switched system in the singular perturbation form

$$
\dot{x}(t)=M^{\sigma(t)}(\varepsilon) x(t),
$$

with $M^{i}(\varepsilon)$ defined in (3) for any $i \in \Xi$. The existence of a quadratic Lyapunov function $V(x, \varepsilon)=x^{\prime} P(\varepsilon) x$ such that $V(x, \varepsilon)>0$ and $\dot{V}(x, \varepsilon)<0$ is a well-known sufficient condition for the asymptotic stability of the system (18). It is equivalent to the following lemma:

Lemma 1: [10] If there exist matrices $P(\varepsilon)=P(\varepsilon)^{\prime} \succ 0$ and $Q^{i}(\varepsilon)=Q^{i}(\varepsilon)^{\prime} \succeq 0$ of appropriate dimension such that

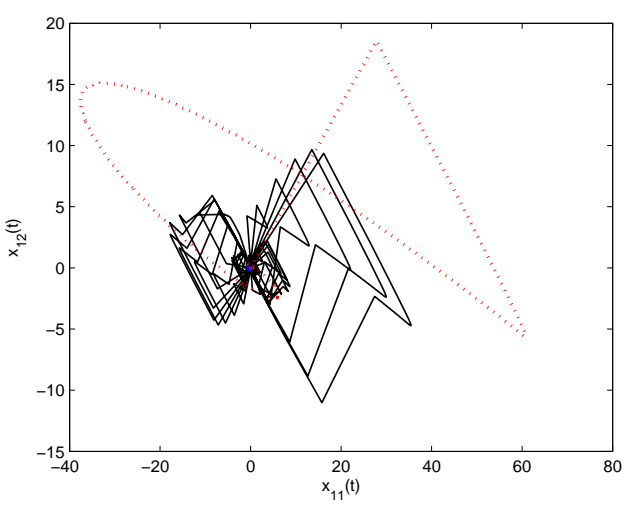

Fig. 3. Fast variables evolution of the system (14): $\sigma(t)=\{1,2,1,2, \ldots\}$ (solid line), $\sigma(t)=\{1\}$ (dashed line), $\sigma(t)=\{2\}$ (dotted line)

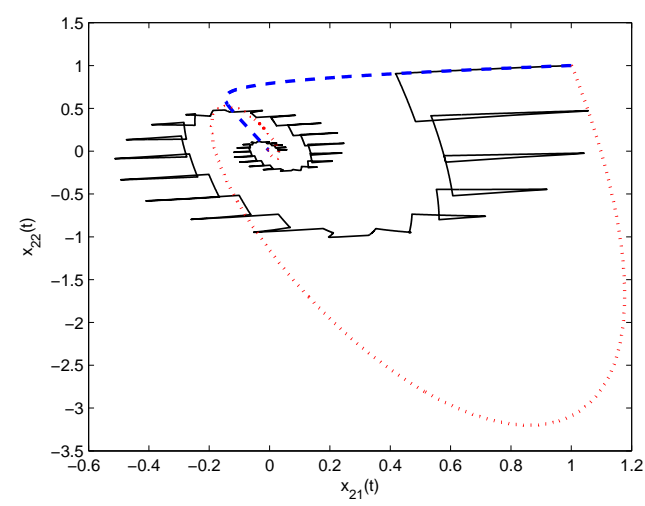

Fig. 4. Slow variables evolution of the system (14): $\sigma(t)=\{1,2,1,2, \ldots\}$ (solid line), $\sigma(t)=\{1\}$ (dashed line), $\sigma(t)=\{2\}$ (dotted line)

\section{LMIs}

$$
M^{i}(\varepsilon) P(\varepsilon)+P(\varepsilon) M^{i}(\varepsilon)^{\prime}+Q^{i}(\varepsilon) \prec 0
$$

are verified $\forall i \in \Xi$, then the switched system (18) is stable.

When $\varepsilon$ is small, the determination of $P(\varepsilon)$ is complicated due to the ill-conditioning of the LMI (19). This problem can be avoided decomposing the two-time scale system into two well-behaved subsystems, the slow and the fast manifolds [8]. The following theorem states LMI conditions which verify the stability of the switched system (18) independently of $\varepsilon$ and for any switching law. The proof, omitted due to space limitations, may be found in [11].

Theorem 1: Assume that there exist matrices $P_{f}=P_{f}^{\prime} \succ$ $0, Q_{f}^{i}=Q_{f}^{i^{\prime}} \succ 0, P_{s}=P_{s}^{\prime} \succ 0, Q_{s}^{i}=Q_{s}^{i^{\prime}} \succ 0$ of appropriate dimension such that LMIs

$$
\begin{gathered}
M_{11}^{i} P_{f}+P_{f} M_{11}^{i{ }^{\prime}}+Q_{f}^{i} \prec 0, \\
M_{s}^{i} P_{s}+P_{s} M_{s}^{i^{\prime}}+Q_{s}^{i} \prec 0, \\
{\left[\begin{array}{cc}
Q_{f}^{i} & -\left(M_{11}^{i} Y^{i}+P_{f} M_{21}^{i}{ }^{\prime}\right) \\
(\star)^{\prime} & Q_{s}^{i}-M_{21}^{i} Y^{i}-Y^{i^{\prime}} M_{21}^{i}{ }^{\prime}
\end{array}\right] \succ 0}
\end{gathered}
$$


are verified $\forall i \in \Xi$, with $Y^{i}=-\sum_{h=1, h \neq i}^{\mathcal{N}} M_{11}^{h}{ }^{-1} M_{12}^{h} P_{s}$. Then, there exists a positive scalar $\varepsilon_{\max }$ such that the switched system (18) is asymptotically stable $\forall \varepsilon \in\left(0, \varepsilon_{\max }\right]$ and for any switching law.

Remark 1: Theorem 1 provides two separate stability conditions for fast and slow manifolds (20) and (21), respectively. Moreover, the coupling condition (22) is given. This allows verifying the classical stability conditions given by Lemma 1 , for any $\varepsilon \in\left(0, \varepsilon_{\max }\right] . P(\varepsilon)$ and $Q^{i}(\varepsilon)$ are defined in (30)-(41), for any $i \in \Xi$.

\section{CONTROL DESIGN}

Consider the linear switched system in the singular perturbation form

$$
\dot{x}(t)=M^{\sigma(t)}(\varepsilon) x(t)+N^{\sigma(t)}(\varepsilon) u(t),
$$

with $M^{i}(\varepsilon)$ and $N^{i}(\varepsilon)$ defined in (3) for any $i \in \Xi$. The aim of this section is to design a state-feedback control law

$$
u(t)=K^{\sigma(t)}(\varepsilon) x(t)
$$

stabilizing the closed-loop system (23) for any switching law.

Lemma 2: If there exist matrices $P(\varepsilon)=P(\varepsilon)^{\prime} \succ 0$, $Q^{i}(\varepsilon)=Q^{i}(\varepsilon)^{\prime} \succeq 0$ and $Z^{i}(\varepsilon)$ of appropriate dimension such that LMIs

$$
\begin{aligned}
& M^{i}(\varepsilon) P(\varepsilon)+P(\varepsilon) M^{i}(\varepsilon)^{\prime}+N^{i}(\varepsilon) Z^{i}(\varepsilon)+ \\
& Z^{i}(\varepsilon)^{\prime} N^{i}(\varepsilon)^{\prime}+Q^{i}(\varepsilon) \prec 0
\end{aligned}
$$

are verified $\forall i \in \Xi$, then the state-feedback control law (24) stabilizes asymptotically the continuous-time switched system (23), with $K^{i}(\varepsilon)=Z^{i}(\varepsilon) P(\varepsilon)^{-1}$.

As in the stability analysis case, when $\varepsilon$ is small some difficulties to compute the gains $K^{i}(\varepsilon)$ arise. This problem is due to the ill-conditioning of the constraint (25) and can be avoided decomposing the two-time scale system into two well-behaved subsystems, the slow and the fast manifolds [8]. The following theorem gives LMI conditions which verify the stability of the system (23) independently of $\varepsilon$, for any switching law.

Theorem 2: Assume that there exist matrices $P_{f}=P_{f}^{\prime} \succ$ $0, Q_{f}^{i}=Q_{f}^{i \prime} \succ 0, Z_{f}^{i}, P_{s}=P_{s}^{\prime} \succ 0, Q_{s}^{i}=Q_{s}^{i^{\prime}} \succ 0$ and $Z_{s}^{i}$ of appropriate dimension such that LMIs

$$
\begin{array}{r}
M_{11}^{i} P_{f}+P_{f} M_{11}^{i}{ }^{\prime}+N_{1}^{i} Z_{f}^{i}+Z_{f}^{i^{\prime}} N_{1}^{i^{\prime}}+Q_{f}^{i} \prec 0, \\
M_{s}^{i} P_{s}+P_{s} M_{s}^{i^{\prime}}+N_{s}^{i} Z_{s}^{i}+Z_{s}^{i^{\prime}} N_{s}^{i^{\prime}}+Q_{s}^{i} \prec 0, \\
{\left[\begin{array}{cccc}
Q_{f}^{i} & F^{i} & N_{1}^{i} Z_{f}^{i} & 0 \\
(\star)^{\prime} & G^{i} & Y^{i^{\prime}} & N_{2}^{i} Z_{f}^{i}+Y^{i^{\prime}} \\
(\star)^{\prime} & (\star)^{\prime} & P_{f} & 0 \\
(\star)^{\prime} & (\star)^{\prime} & (\star)^{\prime} & P_{f}
\end{array}\right] \succ 0}
\end{array}
$$

are verified $\forall i \in \Xi$, with $Y^{i}=-\sum_{h=1, h \neq i}^{\mathcal{N}} M_{11}^{h-1}\left(M_{12}^{h} P_{s}+\right.$ $\left.N_{1}^{h} Z_{s}^{h}\right), F^{i}=-\left(M_{11}^{i} Y^{i}+P_{f} M_{21}^{i}{ }^{\prime}+Z_{f}^{i^{\prime}} N_{2}^{i^{\prime}}\right)$ and $G^{i}=$
$Q_{s}^{i}-M_{21}^{i} Y^{i}-Y^{i^{\prime}} M_{21}^{i}{ }^{\prime}$. Then, there exists a positive scalar $\varepsilon_{\max }$ such that the state-feedback controller gains

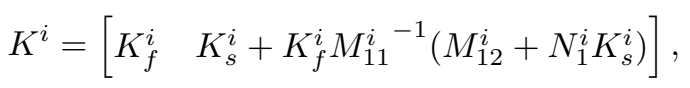

with $K_{f}^{i}=Z_{f}^{i} P_{f}^{-1}$ and $K_{s}^{i}=Z_{s}^{i} P_{s}^{-1}$, asymptotically stabilize the closed-loop switched system (23), $\forall \varepsilon \in\left(0, \varepsilon_{\max }\right]$ and for any switching law.

Proof: Let us assume

$$
\begin{gathered}
P(\varepsilon)=\left[\begin{array}{ll}
P_{1}(\varepsilon) & P_{2}(\varepsilon) \\
P_{2}(\varepsilon)^{\prime} & P_{3}(\varepsilon)
\end{array}\right] \succ 0, \\
Z^{i}(\varepsilon)=\left[\begin{array}{ll}
Z_{1}^{i}(\varepsilon) & Z_{2}^{i}(\varepsilon)
\end{array}\right], \\
Q^{i}(\varepsilon)=\left[\begin{array}{ll}
Q_{1}^{i}(\varepsilon) & Q_{2}^{i}(\varepsilon) \\
Q_{2}^{i}(\varepsilon)^{\prime} & Q_{3}^{i}(\varepsilon)
\end{array}\right] \succ 0,
\end{gathered}
$$

with

$$
P_{1}(\varepsilon)=P_{f}+\varepsilon P_{2} P_{s}^{-1} P_{2}^{\prime}
$$

$$
\begin{gathered}
P_{2}(\varepsilon)=\varepsilon P_{2}=-\varepsilon \sum_{h=1}^{\mathcal{N}} M_{11}^{h}{ }^{-1}\left(M_{12}^{h} P_{s}+N_{1}^{h} Z_{s}^{h}\right), \\
P_{3}(\varepsilon)=\varepsilon P_{s} \\
Z_{1}^{i}(\varepsilon)=Z_{f}^{i}+\varepsilon Z_{s}^{i} P_{s}^{-1} P_{2}^{\prime} \\
Z_{2}^{i}(\varepsilon)=\varepsilon\left(Z_{s}^{i}+Z_{f}^{i} P_{f}^{-1} Y^{i}\right) \\
Q_{1}^{i}(\varepsilon)=\varepsilon^{-1} Q_{f}^{i}
\end{gathered}
$$

$Q_{2}^{i}(\varepsilon)=-\left(\left(M_{11}^{i}+N_{1}^{i} Z_{f}^{i} P_{f}^{-1}\right) Y^{i}+P_{f} M_{21}^{i}{ }^{\prime}+Z_{f}^{i^{\prime}} N_{2}^{i^{\prime}}\right)$,

$$
\begin{array}{r}
Q_{3}^{i}(\varepsilon)=\varepsilon\left(Q_{s}^{i}-\left(M_{21}^{i}+N_{2}^{i} Z_{f}^{i} P_{f}^{-1}\right) Y^{i}-\right. \\
\left.Y^{i^{\prime}}\left(M_{21}^{i}{ }^{\prime}+P_{f}^{-1} Z_{f}^{i^{\prime}} N_{2}^{i^{\prime}}\right)\right)
\end{array}
$$

and

$$
Y^{i}=-\sum_{h=1, h \neq i}^{\mathcal{N}} M_{11}^{h-1}\left(M_{12}^{h} P_{s}+N_{1}^{h} Z_{s}^{h}\right) .
$$

Substituting (3) and (30)-(32) in (25), we have:

$$
\left[\begin{array}{ll}
X_{1}^{i}(\varepsilon) & X_{2}^{i}(\varepsilon) \\
X_{2}^{i}(\varepsilon)^{\prime} & X_{3}^{i}(\varepsilon)
\end{array}\right]
$$

with $X_{1}^{i}(\varepsilon), X_{2}^{i}(\varepsilon)$ and $X_{3}^{i}(\varepsilon)$ defined in the next page (equations (43)-(45)). Replacing the values of $P(\varepsilon), Z^{i}(\varepsilon), Q^{i}(\varepsilon)$ and the equations (6), (33)-(41), we obtain the equations (46)-(48) (see the next page).

The equation (42) can be written as

$$
\left[\begin{array}{cc}
\varepsilon^{-1}\left(X_{f}^{i}+O(\varepsilon)\right) & \varepsilon\left(X_{2}^{i}+O(\varepsilon)\right) \\
(\star)^{\prime} & \varepsilon\left(X_{s}^{i}+O(\varepsilon)\right)
\end{array}\right] .
$$

For assumption $X_{f}^{i} \prec 0$ and $X_{s}^{i} \prec 0$. This means that there exists a scalar $\varepsilon_{\max }>0$ such that $X_{s}^{i}+O(\varepsilon) \prec 0$ and $X_{f}^{i}-\varepsilon^{2} X_{2}^{i} X_{s}^{i^{-1}} X_{2}^{i^{\prime}}+O(\varepsilon) \prec 0, \forall i \in \Xi$ and $\forall \varepsilon \in\left(0, \varepsilon_{\max }\right]$. Hence, using the Schur complement, the LMI (25) is verified. Since $P_{f} \succ 0$ and $P_{s} \succ 0$, the LMI (30) holds. Furthermore, substituting (38)-(40) in (32), we obtain the LMI (50) which, using the Schur complement, 


$$
\begin{gathered}
X_{1}^{i}(\varepsilon)=\varepsilon^{-1}\left(M_{11}^{i} P_{1}(\varepsilon)+P_{1}(\varepsilon) M_{11}^{i}{ }^{\prime}+M_{12}^{i} P_{2}(\varepsilon)^{\prime}+P_{2}(\varepsilon) M_{12}{ }^{\prime}+N_{1}^{i} Z_{1}^{i}(\varepsilon)+Z_{1}^{i}(\varepsilon)^{\prime} N_{1}^{i^{\prime}}+Q_{1}^{i}(\varepsilon)\right), \\
X_{2}^{i}(\varepsilon)=\varepsilon^{-1} M_{11}^{i} P_{2}(\varepsilon)+\varepsilon^{-1} M_{12}^{i} P_{3}(\varepsilon)+P_{1}(\varepsilon) M_{21}^{i}{ }^{\prime}+P_{2}(\varepsilon)^{\prime} M_{22}^{i}{ }^{\prime}+\varepsilon^{-1} N_{1}^{i} Z_{2}^{i}(\varepsilon)+Z_{1}^{i}(\varepsilon)^{\prime} N_{2}^{i^{\prime}}+Q_{2}^{i}(\varepsilon), \\
X_{3}^{i}(\varepsilon)=M_{22}^{i} P_{3}(\varepsilon)+P_{3}(\varepsilon) M_{22}^{i}{ }^{\prime}+M_{21}^{i} P_{2}(\varepsilon)+P_{2}(\varepsilon)^{\prime} M_{21}^{i}{ }^{\prime}+N_{2}^{i} Z_{2}^{i}(\varepsilon)+Z_{2}^{i}(\varepsilon)^{\prime} N_{2}^{i^{\prime}}+Q_{3}(\varepsilon) .
\end{gathered}
$$

$$
\begin{gathered}
X_{1}^{i}(\varepsilon)=\varepsilon^{-1}\left(M_{11}^{i} P_{f}+P_{f} M_{11}^{i}{ }^{\prime}+N_{1}^{i} Z_{f}^{i}+Z_{f}^{i^{\prime}} N_{1}^{i^{\prime}}+Q_{f}^{i}+O(\varepsilon)\right)=\varepsilon^{-1}\left(X_{f}^{i}+O(\varepsilon)\right), \\
X_{2}^{i}(\varepsilon)=\varepsilon\left(P_{2}^{\prime} M_{22}^{i}{ }^{\prime}+O(\varepsilon)\right)=\varepsilon\left(X_{2}^{i}+O(\varepsilon)\right), \\
X_{3}^{i}(\varepsilon)=\varepsilon\left(M_{s}^{i} P_{s}+P_{s} M_{s}^{i^{\prime}}+N_{s}^{i} Z_{s}^{i}+Z_{s}^{i^{\prime}} N_{s}^{i^{\prime}}+Q_{s}^{i}+O(\varepsilon)\right)=\varepsilon\left(X_{s}^{i}+O(\varepsilon)\right) .
\end{gathered}
$$

$$
Q^{i}(\varepsilon)=\left[\begin{array}{cc}
\varepsilon^{-1} I_{n_{1}} & 0 \\
0 & I_{n_{2}}
\end{array}\right]\left[\begin{array}{cc}
Q_{f}^{i} & -\left(\left(M_{11}^{i}+N_{1}^{i} Z_{f}^{i} P_{f}^{-1}\right) Y^{i}+P_{f} M_{21}^{i}{ }^{\prime}+Z_{f}^{i{ }^{\prime}} N_{2}^{i^{\prime}}\right) \\
(\star)^{\prime} & Q_{s}^{i}-\left(M_{21}^{i}+N_{2}^{i} Z_{f}^{i} P_{f}^{-1}\right) Y^{i}-Y^{i^{\prime}}\left(M_{21}^{i}{ }^{\prime}+P_{f}^{-1} Z_{f}^{i^{\prime}} N_{2}^{i^{\prime}}\right)
\end{array}\right]\left[\begin{array}{cc}
I_{n_{1}} & 0 \\
0 & \varepsilon I_{n_{2}}
\end{array}\right] \succ 0
$$

$$
\left[\begin{array}{cccc}
Q_{f}^{i}+N_{1}^{i} Z_{f}^{i} P_{f}^{-1} Z_{f}^{i \prime} N_{1}^{i^{\prime}} & -\left(M_{11}^{i} Y^{i}+P_{f} M_{21}^{i}{ }^{\prime}+Z_{f}^{i^{\prime}} N_{2}^{i^{\prime}}\right) & N_{1}^{i} Z_{f}^{i} & 0 \\
(\star)^{\prime} & Q_{s}^{i}-M_{21}^{i} Y^{i}-Y^{i^{\prime}} M_{21}^{i}{ }^{\prime}+N_{2}^{i} Z_{f}^{i} P_{f}^{-1} Z_{f}^{i^{\prime}} N_{2}^{i^{\prime}}+Y^{i^{\prime}} P_{f}^{-1} Y^{i} & Y^{i^{\prime}} & N_{2}^{i} Z_{f}^{i}+Y^{i^{\prime}} \\
(\star)^{\prime} & (\star)^{\prime} & P_{f} & 0 \\
(\star)^{\prime} & (\star)^{\prime} & (\star)^{\prime} & P_{f}
\end{array}\right] \succ 0
$$

is verified if and only if (50) holds. (28) is no negative definite. This implies that the constraint (51) holds $\forall i \in \Xi$. Thus, also LMI (32) holds. Equations (50) and (51) are defined in the next page.

In order to find $K^{i}$, consider

$$
u_{s}(t)=K_{s}^{i} x_{s}(t)=Z_{s}^{i} P_{s}^{-1} x_{s}(t)
$$

and

$$
u_{f}(t)=K_{f}^{i} x_{f}(t)=Z_{f}^{i} P_{f}^{-1} x_{f}(t) .
$$

The composite controller is

$$
u_{c}(t)=u_{s}(t)+u_{f}(t)=K_{s}^{i} x_{s}(t)+K_{f}^{i} x_{f}(t) .
$$

Letting $x_{s}(t)=x_{2}(t)$ and $x_{f}(t)=x_{1}(t)+$ $M_{11}^{i}{ }^{-1}\left(M_{12}^{i} x_{s}(t)+N_{1}^{i} u_{s}(t)\right)=x_{1}(t)+M_{11}^{i}{ }^{-1}\left(M_{12}^{i}+\right.$ $\left.N_{1}^{i} K_{s}^{i}\right) x_{s}(t)$, we have

$$
\begin{aligned}
u_{c}(t)= & Z_{s}^{i} P_{s}^{-1} x_{2}(t)+Z_{f}^{i} P_{f}^{-1} x_{1}(t)+ \\
& Z_{f}^{i} P_{f}^{-1} M_{11}^{i}{ }^{-1}\left(M_{12}^{i}+N_{1}^{i} Z_{s}^{i} P_{s}^{-1}\right) x_{2}(t) .
\end{aligned}
$$

When $\varepsilon \rightarrow 0$, substituting (30) and (31) in $K^{i}(\varepsilon)=$ $Z(\varepsilon)^{i} P(\varepsilon)^{-1}$ and applying the formula of the inverse of partitioned matrix, we find (55), which concludes the proof.

Remark 2: LMI conditions of Theorem 2 with $Z_{f}^{i}=0$ lead to the reduced control law

$$
u(t)=K_{r}^{i}\left[\begin{array}{l}
x_{1}(t) \\
x_{2}(t)
\end{array}\right]=\left[\begin{array}{ll}
0 & K_{s}^{i}
\end{array}\right]\left[\begin{array}{l}
x_{1}(t) \\
x_{2}(t)
\end{array}\right]
$$

where $K_{s}^{i}=Z_{s}^{i} P_{s}^{-1}$ are the controller gain of the slow subsystem and stabilize asymptotically the switched system (23) $\forall i \in \Xi$ and $\forall \varepsilon \in\left(0, \varepsilon_{\max }\right]$.

Notice that in this case (26) implies that the fast subsystem must be asymptotically stable in open loop.

Remark 3: The conditions given in Theorem 2 are the design version of the conditions proposed in Theorem 1 . Thus, conditions of Theorem 1 can be recovered setting $Z_{f}^{i}=Z_{s}^{i}=0$ for any $i \in \Xi$.

\section{NUMERICAL EXAMPLE}

Let a continuous-time switched system in the singular perturbation form (4), with $\Xi=\{1,2\}, \varepsilon=0.005$ and

$$
\begin{aligned}
& M_{11}^{1}=\left[\begin{array}{cc}
0 & 1 \\
-1 & -2
\end{array}\right], M_{12}^{1}=\left[\begin{array}{cc}
0 & 0 \\
1.5 & 0
\end{array}\right], \\
& M_{21}^{1}=\left[\begin{array}{cc}
0 & 0 \\
-0.6 & -0.5
\end{array}\right], M_{22}^{1}=\left[\begin{array}{cc}
0 & 1 \\
2.1 & 0
\end{array}\right], \\
& N_{1}^{1}=\left[\begin{array}{c}
0 \\
-1
\end{array}\right], N_{2}^{1}=\left[\begin{array}{l}
0 \\
0
\end{array}\right], \\
& M_{11}^{2}=\left[\begin{array}{cc}
0 & 1 \\
-3 & -5
\end{array}\right], M_{12}^{2}=\left[\begin{array}{ll}
0 & 0 \\
0 & 0
\end{array}\right], \\
& M_{21}^{2}=\left[\begin{array}{cc}
0 & 0 \\
-0.3 & -0.2
\end{array}\right], M_{22}^{2}=\left[\begin{array}{cc}
0 & 0.7 \\
0 & 0
\end{array}\right], \\
& N_{1}^{2}=\left[\begin{array}{c}
0 \\
-1
\end{array}\right], N_{2}^{2}=\left[\begin{array}{l}
0 \\
0
\end{array}\right] .
\end{aligned}
$$




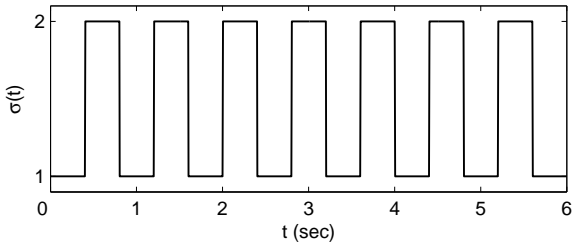

Fig. 5. Switching law $\sigma(t)$
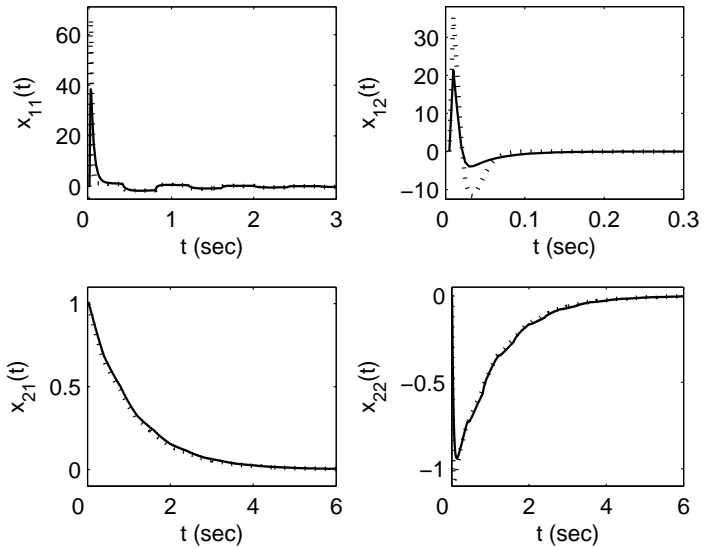

Fig. 6. Closed-loop response in the continuous-time with full state-feedback controller (solid line) and reduced state-feedback controller (dotted line)

The subsystem 1 is open loop unstable and the subsystem 2 is characterized by a state-space matrix with zero eigenvalues. Theorem 2 leads to the following controller gains:

$$
\begin{aligned}
K^{1} & =\left[\begin{array}{llll}
0.4040 & 0.1511 & -65.3601 & -60.3074
\end{array}\right], \\
K^{2} & =\left[\begin{array}{llll}
-0.4110 & -0.5931 & -147.6057 & -137.0206
\end{array}\right] .
\end{aligned}
$$

Since $M_{11}^{1}$ and $M_{11}^{2}$ are Hurwitz, a reduced control law may also be proposed:

$$
\begin{aligned}
& K_{r}^{1}=\left[\begin{array}{llll}
0 & 0 & -99.0779 & -88.5710
\end{array}\right], \\
& K_{r}^{2}=\left[\begin{array}{llll}
0 & 0 & -347.0992 & -310.4213
\end{array}\right] .
\end{aligned}
$$

Consider the switching law given in Fig. 5 and the initial condition $x(0)=\left[\begin{array}{llll}0 & 0 & 1 & 0\end{array}\right]^{\prime}$, Fig. 6 shows the results, with $x(t)=\left[\begin{array}{llll}x_{11}(t) & x_{12}(t) & x_{21}(t) & x_{22}(t)\end{array}\right]^{\prime}$. The solid line represents the state variables evolution using the full state-feedback controller gains $K^{1}$ and $K^{2}$ while the dotted line represents the state variables evolution using the reduced state-feedback controller gains $K_{r}^{1}$ and $K_{r}^{2}$. Fig. 7 shows the control signal evolution.

\section{CONCLUSION}

In this article, asymptotic stability of two-time scale switched systems was investigated. We showed that asymptotic stability of slow and fast switched subsystems does not imply the asymptotic stability of the corresponding two-time scale switched systems in the singular perturbation form. A coupling constraint must also be considered. Hence, we presented LMI conditions to assess asymptotic stability of two-time scale switched systems under an arbitrary switching

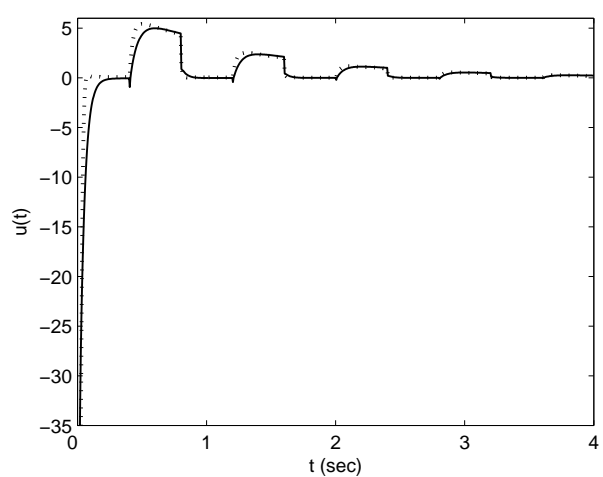

Fig. 7. Control signal evolution in the continuous-time with full statefeedback controller (solid line) and reduced state-feedback controller (dotted line)

law. For the same class of systems, we also provided statefeedback control design. A numerical example shows the validity of our approach.

\section{REFERENCES}

[1] M. Alwan, X. Liu, and B. Ingalls. Exponential stability of singularly perturbed switched systems with time delay. Nonlinear Analysis: Hybrid Systems, 2(3):913-921, 2008.

[2] S. Branicky. Multiple Lyapunov function and other analysis tools for switched and hybrid systems. IEEE Transactions on Automatic Control, 43(4):475-482, 1998.

[3] W. P. Dayawansa and C. F. Martin. A converse Lyapunov theorem for a class of dynamical systems which undergo switching. IEEE Transactions on Automatic Control, 44(4):751-760, 1999.

[4] R. A. Decarlo, M. S. Branicky, S. Pettersson, and B. Lennartson. Perspectives and results on the stability and stabilizability ofhybrid systems. Proceedings of the IEEE, 88(7):1069-1082, 2000.

[5] G. Garcia, J. Daafouz, and J. Bernussou. The infinite time near optimal decentralized regulator problem for singularly perturbed systems: a convex optimization approach. Automatica, 38(8):1397-1406, 2002.

[6] J. Hespanha and A. S. Morse. Stability of switched systems with average dwell-time. In Conf. on Decision and Control, 1999.

[7] P. Kokotovic. A Riccati equation for block-diagonalization of illconditioned systems. IEEE Transactions on Automatic Control, 20(6):812- 814, 1975.

[8] P. Kokotovic, H.K. Khalil, and J. O'Reilly. Singular perturbation methods in control: analysis and design. Academic Press, 1986.

[9] P. Kokotovic and P. Sannuti. Singular perturbation method for reducing the model order in optimal control design. IEEE Transactions on Automatic Control, 13(4):377-384, 1968.

[10] D. Liberzon. Switching in systems and control. Birkhäuser, 2003.

[11] I. Malloci. Two time scale switched systems: Application to steering control in hot strip mills. PhD Thesis, CRAN UMR 7039 - Nancy University, CNRS, 2009.

[12] I. Malloci, J. Daafouz, C. Iung, R. Bonidal, and P. Szczepanski. Switched system modeling and robust steering control of the tail end phase in a hot strip mill. Nonlinear Analysis: Hybrid Systems, 3(3):239-250, 2009.

[13] A.S. Morse. Supervisory control of families of linear set-point controllers - part 1: Exact matching. IEEE Transactions on Automatic Control, 41:1413-1431, 1996.

[14] D.S. Naidu. Singular perturbations and time scales in control theory and applications: An overview. Dynamics of Continuous, Discrete \& Impulsive Systems. Series B. Applications \& Algorithms, 9(2):233278, 2002.

[15] R. Shorten, F. Wirth, O. Mason, K. Wulff, and C. King. Stability criteria for switched and hybrid systems. SIAM Review, 49(4):545592, 2007.

[16] G. Zhai, B. Hu, K. Yasuda, and A.N. Michel. Stability analysis of switched systems with stable and unstable subsystems: An average dwell time approach. International Journal of Systems Science, 32(8):1055 -1061, 2001. 